\title{
Antidiabetics in Combination with Hydroxychloroquine Improve Antioxidant and Hepatoprotective Activities in Alloxan-Induced Diabetic Rats
}

\author{
Shaheda Zannah ${ }^{1}$, Monirul Islam ${ }^{2}$, Yusuf Ali ${ }^{1}$, Md. Asaduzzaman ${ }^{1}$ Md. Shahid Sarwar ${ }^{1}$, \\ Abdul Alim Al Bari ${ }^{2}$ and Mamunur Rashid ${ }^{1,2}$
}

${ }^{1}$ Department of Pharmacy, Southeast University, Dhaka-1213, Bangladesh

${ }^{2}$ Department of Pharmacy, Rajshahi University, Rajshahi-6205, Bangladesh

Received: November 22, 2014; Accepted: January 30, 2015

\begin{abstract}
Hyperglycemia exerts toxic effects on the pancreatic $\beta$-cells. This study investigated the hypothesis that the antidiabetic drugs glibenclamide and metformin, in combination with hydroxychloroquine (HCQ) offer additional protection for the pancreas against oxidative stress and produce hepatoprotective effect in alloxan-induced diabetic rats. Diabetes was induced in male Long-Evans rats by a single dose of alloxan (120 mg/kg; i.p.). Different groups of diabetic animals were treated with glibenclamide $(10 \mathrm{mg} / 70 \mathrm{~kg}$, i.p. $)$, metformin $(850 \mathrm{mg} / 70 \mathrm{~kg}$, i.p. $)$, HCQ (300 mg/70 kg, i.p.) and combination of both glibenclamide and metformin with HCQ, separately for a period of 28 days. Diabetic rats had significantly elevated levels of serum glutamate oxaloacetate transaminase (SGOT) and serum glutamate pyruvate transaminase (SGPT), while catalase (CAT) and superoxide dismutase (SOD) activity were significantly reduced. Glibenclamide and metformin produced no significant effects on antioxidant enzymes but both showed significant $(\mathrm{p}<0.05)$ result in reducing SGOT and SGPT level in diabetic rats. In contrast, the combination of glibenclamide or metformin with HCQ showed better effect on up-regulation of CAT and SOD activity and down-regulation of SGOT and SGPT activity in comparison with the antidiabetic drug alone. These findings suggest that, HCQ potentiates the effect of glibenclamide and metformin to protect pancreas against oxidative stress and produce hepatoprotective effect in diabetic rats.
\end{abstract}

Key words: Combination, Hepatoprotective Activity, Hydroxychloroquine, Glibenclamide, Metformin.

\section{Introduction}

Diabetes mellitus is associated with progressive metabolic derangement, worsening glycemic control and morphological changes in the kidney, retina, pancreas and other organs (Soret et al., 1974; Cristina et al., 2008; Cook et al., 2005). Oxidative stress is known to play a significant role in the induction of these processes (Giugliano et al., 1996; Baynes and Thorpe, 1999). High levels of oxidative stress with excessive generation of free radicals and depleted levels of free radicals scavenging enzymes have been demonstrated in several studies, both in experimental animal models of diabetes and in human diabetic subjects (Bonnefont-Rousselot et al., 2000; Telci et al., 2000; Turk et al., 2002). In type 1 diabetes, reactive oxygen species (ROS) are involved in $\beta$-cell dysfunction initiated by autoimmune reactions and inflammatory cytokines (Cnop et al., 2005). In type 2 diabetes, ROS activate $\beta$-cell apoptotic pathways, impair insulin synthesis and also contribute to insulin resistance (Evans et al., 2003; Simmons, 2006). While oral hypoglycemic agents may be effective for glycemic control, at least in the early stages of diabetes, they do not appear to be effective in entirely preventing the progression of ROS mediated organ damage (UKPDS, 1998). Hepatic abnormalities such as elevations of transaminases and alkaline phosphatase (ALP) are common in diabetes mellitus (Erbey et al., 2000; Vozarova et al., 2002; Meybodi et al., 2008; Leeds et al., 2009). The measurement of these enzymes and other biochemical markers such as albumin, total bilirubin in serum constitutes the liver function tests. These liver function tests are frequently utilized to diagnose or screen for hepatobiliary disease, examine the progression of a disease as well as to monitor or detect the hepatotoxicity

Correspondence to: Mamunur Rashid; E-mail: mamun69jp@yahoo.com 
that may arise from the use of drugs or substances (Elizabeth et al., 2005; Senior et al., 2009). Measurement of serum aspartate aminotransferase (AST) and alanine aminotransferase (ALT) reflects the concentrations of intracellular AST and ALT that have leaked into the general circulation and thus, serves as an indicator of hepatotoxicity (Elizabeth et al., 2005). In our previous study, we have reported that HCQ in combination with glibenclamide or metformin exerts hypoglycemic and antidyslipidemic effects in alloxan-induced diabetic rats (Zannah et al., 2014). We have also showed that oral hypoglycemic agents (metformin and/or glibenclamide) combined with HCQ improves glycemic control and are more effective in ameliorating liver glycogen level in diabetic rats than either drug alone. The main aim of this study was to investigate the potential hepatoprotective effect of HCQ combined with glibenclamide or metformin in alloxan-induced diabetic rats by measuring the activities of serum AST and ALT and the effect on oxidative stress by measuring antioxidant enzyme activities like SOD and CAT. In the present study, we have investigated how the addition of HCQ as an adjunct to glibenclamide or metformin affects hepatoprotective and oxidative stress in alloxan-induced diabetic rats (AIDRs).

\section{Materials and Methods}

Selection of animal: Long-Evans male rats weighing about 150-180 gm, age 2 months were acclimatized to the new environmental condition for a period of one week. During the experimental period, the rats were kept in a well ventilated animal house at room temperature of $25^{\circ} \mathrm{C}$ and were supplied with standard pellets from ICDDR, B and fresh drinking water. All the rats were kept in cages and maintained with natural 12 hour light and dark cycle.

Experimental induction of diabetes: Long-Evans male rats except normal control group were allowed to fast for 12 hours. Hyperglycemia was induced in each fasted rat by administering alloxan monohydrate $(120 \mathrm{mg} / \mathrm{Kg}$ body weight) in normal saline intraperitoneally. $10 \%$ dextrose was thereafter administered orally to combat the immediate hypoglycemia that could occur. After 24 hours, blood glucose level was measured by using Clever Check glucose test meter (Bioland, Germany) using blood sample collected from the tail vein of the rats. When the condition of diabetes was established, animals with blood glucose levels above $11.1 \mathrm{mmol} / \mathrm{L}$ were selected for the study.

Preparation of HCQ, glibenclamide and metformin: Two hypoglycemic drugs (metformin \& glibenclamide) were used in the present study as reference standard drug. Each drug was administered intraperitoneally to hyperglycaemic rats $(n=6)$. Metformin was administered at $850 \mathrm{mg} / 70 \mathrm{~kg}$ body weight/day and glibenclamide at 10 $\mathrm{mg} / 70 \mathrm{~kg}$ body weight/day. The trial drug hydroxychloroquine was administered at $300 \mathrm{mg} / 70 \mathrm{~kg}$ body weight/day. Glibenclamide and metformin were dissolved in dimethyl sulfoxide and distilled water respectively before they were administered. HCQ was dissolved in distilled water. Incase of combination therapy, metformin with HCQ was prepared in solution form according to the dose of $(425 \mathrm{mg} / 70 \mathrm{~kg} \mathrm{BW}+150$ $\mathrm{mg} / 70 \mathrm{~kg} \mathrm{BW})$ and glibenclamide with HCQ was prepared according to the dose of $(5 \mathrm{mg} / 70 \mathrm{~kg} \mathrm{BW}+150 \mathrm{mg} / 70 \mathrm{~kg}$ $\mathrm{BW})$ respectively.

Treatment of animals: The animals were randomly divided into seven groups. Each group comprised of six rats. The treatment of animals began on the 3rd day after alloxan injection and this was considered as 1st day of treatment. The animals were treated for 4 weeks as follows:

-Group 1: Normal control + Distilled water $(0.5 \mathrm{ml})$

-Group 2: Diabetic control + Distilled water $(0.5 \mathrm{ml})$

-Group 3: Diabetic + HCQ (300 mg/70 kg body weight)

-Group 4: Diabetic + Glibenclamide $(10 \mathrm{mg} / 70 \mathrm{~kg}$ body weight)

-Group 5: Diabetic + Glibenclamide $(5 \mathrm{mg} / 70 \mathrm{~kg}$ body weight $)+$ HCQ (150 mg/70 kg body weight)

-Group 6: Diabetic + Metformin (850 mg/70 kg body weight)

-Group 7: Diabetic + Metformin (425 mg/70 kg body weight) + HCQ (150 mg/70 kg body weight)

At the end of the treatment period, the animals were fasted for at least 16 hours and sacrificed. Blood samples were collected in centrifuge tubes without anticoagulants and allowed to clot. The clotted blood was then centrifuged at $4000 \mathrm{rpm}$ for $30 \mathrm{~min}$. Serum was separated and then quickly stored at refrigerator for biochemical analysis. 
Experimental design: Glibenclamide, metformin, HCQ and combinations (glibenclamide with HCQ) and (metformin with HCQ) were administered daily for four weeks in the alloxan-induced diabetic rats. After four weeks treatment antioxidant enzymes like SOD \& CAT activity and liver dysfunction indices like SGOT \& SGPT levels were measured using diagnostic kits.

Hepatoprotective activity test: The blood serum which was collected from thoracic artery after sacrificing the rats was used for testing the serum SGPT (ALT) and SGOT (AST) levels. The concentrations were analyzed by taking absorbance by UV spectrophotometer, using diagnostic kits (Linear Chemicals, Spain).

Evaluation of super oxide dismutase (SOD) and catalase (CAT) enzyme activity: After the experimental period, the animals were sacrificed; liver was isolated and homogenized in chilled Tris buffer at a concentration of $10 \%(\mathrm{w} / \mathrm{v})$. The homogenate was centrifuged at $4000 \mathrm{rpm}$ for 15 minutes in cold centrifuge and supernatant was assayed for SOD and CAT activity by our previously published method (Begum et. al., 2014).

Statistical analysis: The results were expressed as mean \pm SEM. We used a one-way analysis of variance (ANOVA), followed by Dunnett's post-hoc test or students paired or unpaired t-test where appropriate. The statistical method applied in each analysis. Results were considered to be significant when $\mathrm{P}$ values were less than $0.05(\mathrm{p}<0.05)$.

\section{Results and Discussion}

The effect of drug alone (metformin/ glibenclamide/ HCQ) and combination (metformin with HCQ and glibenclamide with $\mathrm{HCQ}$ ) on the parameters of liver dysfunction indices like SGOT \& SGPT levels and antioxidant enzyme activity like SOD \& CAT were performed for long term alloxan-induced diabetic rats.

\section{Effect on liver dysfunction indices}

Effect on SGOT Level: After four weeks treatment, diabetic control group showed a significant increase $(\mathrm{p}<0.01)$ in SGOT levels $(125.55 \%)$ as compared to normal control group. Administration of glibenclamide and metformin to diabetic animals significantly $(\mathrm{p}<0.05)$ declined the SGOT levels as compared to alloxan treated diabetic animals (Table 1). Combination of glibenclamide or metformin with HCQ further reduced the SGOT level in diabetic rats. Moreover, the effect of the combination treatment on SGOT level was significantly $(p<0.01)$ more than that of glibenclamide and metformin. No significant $(\mathrm{p}<0.05)$ effect was observed with HCQ alone.

Effect on SGPT level: Alloxan administration to animals of diabetic control group resulted in a significant increase $(\mathrm{p}<0.01)$ in SGPT levels $(106.17 \%)$ after four weeks treatment when compared with normal control rats. SGPT levels of animals treated with metformin and glibenclamide showed significant decrease $(\mathrm{p}<0.05)$ when compared to alloxan treated animals. On the other hand, the diabetic rats that received a combination of glibenclamide or metformin with HCQ showed further significant $(\mathrm{p}<0.01)$ decrease in SGPT level when compared with alloxan-induced diabetic rats as depicted in (Table 1). Moreover, the effect of the combination treatment on the SGPT level was significantly $(\mathrm{p}<0.01)$ more than that achieved by the glibenclamide and metformin alone. No significant $(p<0.05)$ effect was observed with HCQ alone.

Table 1. Effect of drugs on SGOT and SGPT levels in AIDRs after four weeks treatment.

\begin{tabular}{|c|c|c|}
\hline Groups & SGOT (units/l) & SGPT (units/l) \\
\hline Normal control & $20.66 \pm 1.52$ & $24.30 \pm 0.95$ \\
\hline Diabetic control & $46.60 \pm 1.58^{\# \#}$ & $50.10 \pm 3.26^{\# \#}$ \\
\hline $\begin{array}{l}\text { Diabetic }+ \\
\text { Glibenclamide }\end{array}$ & $29.66 \pm 1.22^{*}$ & $33.20 \pm 1.38^{*}$ \\
\hline Diabetic + Metformin & $32.30 \pm 0.54^{*}$ & $35.30 \pm 0.76^{*}$ \\
\hline Diabetic + HCQ & $42.10 \pm 0.99$ & $45.40 \pm 1.02$ \\
\hline $\begin{array}{l}\text { Diabetic }+ \\
\text { Glibenclamide + HCQ }\end{array}$ & $24.80 \pm 0.86^{* *}$ & $27.50 \pm 0.99^{* *}$ \\
\hline $\begin{array}{l}\text { Diabetic + Metformin } \\
+ \text { HCQ }\end{array}$ & $25.90 \pm 2.58^{* *}$ & $29.90 \pm 1.22^{* *}$ \\
\hline
\end{tabular}

\section{Effect on antioxidant enzyme activities}

Effect on SOD activity: The effect of HCQ, glibenclamide, metformin and their combination are presented in Table 2. The activity of SOD was significantly $(\mathrm{p}<0.05)(37.59 \%)$ declined in the diabetic control rats compared with normal control rats. Daily treatment with glibenclamide \& metformin enhanced SOD level by $28.13 \%$ and $24.04 \%$ respectively as compared with diabetic control rats. Combination of HCQ with 
glibenclamide and metformin significantly $(\mathrm{p}<0.01)$ increased SOD level by $54.73 \%$ and $50 \%$ respectively when compared with alloxan-induced diabetic control rats. Combination of HCQ with glibenclamide or metformin produced a significant $(\mathrm{p}<0.01)$ increase in the SOD activity which is higher than that produced by either drug alone $(p>0.05)$. On the other hand, no significant result was observed with HCQ treatment.

Effect on CAT activity: A significant $(\mathrm{p}<0.05$, $48.27 \%$ ) decline in CAT activity was found with diabetic control group when compared with normal control group after four weeks treatment. Combination of HCQ with glibenclamide and metformin showed a significant improvement $(\mathrm{p}<0.01)$ in the CAT activity after 28 days as compared to alloxan-induced diabetic rats (Table 2). Daily treatment with glibenclamide \& metformin enhanced CAT level by $28 \%$ and $25.33 \%$ respectively. Metformin and glibenclamide enhanced only slightly the activity of CAT $(\mathrm{p}>0.05)$ in comparison with diabetic control group. Combination of HCQ with glibenclamide or metformin produced a more significant $(\mathrm{p}<0.01)$ increment in CAT activity which is higher than that produced by either drug alone. Not much difference was observed with HCQ treatment when compared with diabetic control group.

Table 2. Effect of drugs on the activities of SOD and CAT in AIDRs after four weeks treatment.

\begin{tabular}{lcc}
\hline Groups & $\begin{array}{c}\text { SOD (U/mg } \\
\text { protein) }\end{array}$ & $\begin{array}{c}\text { CAT (U/mg } \\
\text { protein) }\end{array}$ \\
\hline Normal control & $6.27 \pm 0.035$ & $29.00 \pm 1.29$ \\
Diabetic control & $3.91 \pm 0.04^{\#}$ & $15.00 \pm 1.16^{\#}$ \\
Diabetic + & $5.01 \pm 0.13$ & $19.20 \pm 1.29$ \\
Glibenclamide & & \\
Diabetic + Metformin & $4.85 \pm 0.135$ & $18.80 \pm 2.03$ \\
Diabetic + HCQ & $4.23 \pm 0.27$ & $16.70 \pm 1.75$ \\
Diabetic + & $6.05 \pm 0.05^{* *}$ & $24.60 \pm 1.42^{* *}$ \\
Glibenclamide + HCQ & & \\
Diabetic + Metformin \\
+ HCQ
\end{tabular}

The present study aims to investigate the effect of coadministration of HCQ with commonly used antidiabetic drugs glibenclamide and metformin for their effect against various biochemical parameters in alloxan-induced diabetes. Alloxan monohydrate is one of the most frequently used inducer of experimental diabetes. It has been shown that the inhibition of mechanism of oxidative phosphorylation in the beta cells is the primary cause of diabetogenic action of alloxan (Bhattacharya, 1955). Alloxan acts by interfering with some essential enzyme/ enzymes of beta cells; which are also present in liver and kidneys in a specific concentration. Therefore, these organs, namely liver and kidneys are also affected by suitable concentrations of alloxan. In the present study, alloxan at the dose of $120 \mathrm{mg} / \mathrm{kg}$ body weight (i.p.) was found to induce diabetes (glucose levels greater than $11.1 \mathrm{mmol} / \mathrm{L}$ ) in 12 hour fasted rats. Our earlier studies have reported a significant antihyperglycemic activity of the antimalarial agent HCQ in combination with two conventional antidiabetic drugs viz. glibenclamide and metformin in alloxan-induced diabetic rats (Zannah et al., 2014). The study also revealed a significant antidiabetic effect of co-administration of HCQ and antidiabetic agents which was found to be comparable to that produced by the administration of $100 \%$ glibenclamide and $100 \%$ metformin alone (Zannah et al., 2014). SGPT is a cytosolic enzyme primarily present in the liver, and SGOT is a mitochondrial enzyme released from heart, liver, skeletal muscles and kidney. SGOT and SGPT are both sensitive markers of hepatocellular injury. When the liver cell is injured or dies, these proteins can leak through the liver cell membrane into the circulation and serum levels will rise. Diabetic rats have been found to be associated with an increase in the activities of SGOT and SGPT. It may be indicator of severe liver and cardiac damage. The higher levels of SGOT and SGPT may give rise to a high concentration of glucose. In other words, the gluconeogenic action of SGOT and SGPT plays the role of providing new supplies of glucose from other sources such as amino acids (Hossain et al., 2011). The present study revealed an increase in both SGPT and SGOT in diabetic animals which indicated damage to vital organs like liver and heart. Treatment with the drugs alone and their combinations helped to restore the SGOT and SGPT levels to normal range. However, the diabetic rats that received glibenclamide and metformin in combination with HCQ showed significant down-regulated activity of SGOT \& SGPT level indicating that glibenclamide and metformin in combination with HCQ provide better attenuation of SGOT and SGPT level than either drug alone. In diabetes mellitus, hyperglycemia can simply 
inactivate antioxidant enzymes such as SOD, CAT and GPX by glycating these proteins and induces oxidative stress which in turn causes lipid peroxidation (Vincent et al., 2004; Kaleem et al., 2006). Decreased antioxidant enzymes levels and enhanced lipids peroxidation have been well documented in alloxan-induced diabetes (Sepici-Dincel et al., 2007; Oyedemi et al., 2011; Monavar et al., 2010). In the enzymatic antioxidant defense system, SOD is one of the important enzymes and scavenges the superoxide radicals by converting them to $\mathrm{H}_{2} \mathrm{O}_{2}$ and molecular oxygen. In this experiment, both the antioxidant enzyme activities have been reduced significantly in alloxan-induced diabetic rats. The observed decrease in SOD activity in diabetic control rats could result from inactivation by $\mathrm{H}_{2} \mathrm{O}_{2}$ or by glycosylation of the enzyme, which have been reported to occur in diabetes. CAT is involved in the elimination of $\mathrm{H}_{2} \mathrm{O}_{2}$ ( $\mathrm{Lin}$ et al., 2005; Soon and Tan, 2002). Based on the findings of the present study, intraperitoneal administration of combination of glibenclamide or metformin with HCQ increased the antioxidant enzymes levels in red blood cells of alloxan-induced diabetic rats. Both the combination therapies showed noticeable improvement in SOD and CAT activities although HCQ alone has milder effects on SOD and CAT activities. Decreased levels of SOD and CAT in the diabetic state may be due to inactivation caused by reactive oxygen species. In treatment groups, the increased CAT activity could be due to higher production of $\mathrm{H}_{2} \mathrm{O}_{2}$. It is possible that CAT activity, which in turn would protect SOD inactivation by $\mathrm{H}_{2} \mathrm{O}_{2}$, and would cause an increase in SOD activity. Increase in SOD activity would protect CAT against inactivation by superoxide anion (Blum et al., 1985). Meformin and glibenclamide used as reference drugs, also show moderate increase in antioxidant enzymes activities which are due to its hypoglycemic effects.

\section{Conclusion}

The findings of this study indicate that HCQ supplementation with antidiabetic drugs in alloxaninduced diabetic rats reduced the levels of SGOT and SGPT as well as increased the levels of antioxidant enzymes like SOD and CAT activities. The administration of commonly prescribed antidiabetic drugs, glibenclamide and metformin in combination with HCQ inhibited oxidative stress and produced hepatoprotective effect in alloxan-induced diabetic rats. In other words, based on our data, it can be said that HCQ provides additional antioxidant and hepatoprotective effect to glibenclamide and metformin. These protections on pancreas and liver might also partially have contributed to the hypoglycemic effect of HCQ in diabetic rats. In addition, it may be suggested that co-administration of HCQ with other therapeutic agents may be effective in minimizing the side effects of oral hypoglycemic drugs. The study concluded that co-administration of glibenclamide or metformin with hydroxychloroquine may prove to be more beneficial in diabetes than either drug alone; but the clinical appropriateness of the combination has still to be confirmed.

\section{Acknowledgement}

The authors are thankful to Square Pharmaceuticals Ltd, Bangladesh for its kind gift of glibenclamide and metformin.

\section{References}

Baynes, J.W. and Thorpe, S.R. 1999. The role of oxidative stress in diabetic complications. Curr. Opin. Endocrinol. 3, $277-$ 284.

Begum, M.M., Rahman, A.F.M.T., Islam, M.S., Asaduzzaman, M., Ali, M.H., Ali, Y., Zannah, S., Alam, A.H.M.K., Rahman, M.A.A. and Rashid, M., 2014. Simvastatin potentiates the antihyperglycemic, antidyslipidimic and antioxidative effect of glibenclamide on alloxan-induced diabetic rats. Pharmacology \& Pharmacy 5, 1059-1069.

Bhattacharya, G. 1955. On the protection against alloxan diabetes by hexoses. Science 121, 427.

Blum, J. and Fridovich, I. 1985. Inactivation of glutathione peroxidase by superoxide radical. Arch. Biochem. Biophys. 240, 500-508

Bonnefont-Rousselot, D., Bastard, J.P., Jaudon, M.C. and Delattre, J. 2000. Consequences of the diabeticstatus on the oxidant / antioxidant balance. Diab. Metab. 26, 163-176.

Cnop, M., Welsh, N., Jonas, J.C., Jörns, A., Lenzen, S. and Eizirik, D.L. 2005. Mechanisms of pancreaticbeta-cell death in type 1 and type 2 diabetes: many differences, few similarities. Diabetes 54, S97-S107.

Cook, M.N., Girman, C.J., Stein, P.P., Alexander, C.M. and Holman, R.R. 2005. Glycemic controlcontinues to deteriorate after sulfonylureas are added to metformin among patients with type 2 diabetes. Diabetes Care $\mathbf{2 8}$, 995-1000

Cristina, L., Roberto, L. and Stefano, D.P. 2008. $\beta$-cell failure in type 2 diabetes mellitus. Curr. Diab. Rep. 8, 179-184. 
Elizabeth, H. and Harris, M.D. 2005. Elevated liver function tests in type 2 diabetes. Clin Diabetes 23,115-119.

Erbey, J.R., Silberman, C. and Lydick, E. 2000. Prevalence of abnormal serum alanine aminotransferase levels in obesepatients and patients with type 2 diabetes. Am. J. Med. 109, 588-590.

Evans, J.L., Goldfine, I.D., Maddux, B.A. and Grodsky, G.M. 2003. Are oxidative stress-activated signalingpathways mediators of insulin resistance and $\beta$-cell dysfunction? Diabetes 52, 1-8.

Giugliano, D., Ceriello, A. and Paolisso, G. 1996. Oxidative stress and diabetic vascular complications. Diabetes Care 19, 257-267.

Hossain, M.S., Ahmed, M. and Islam, A. 2011. Hypolipidemic and hepatoprotective effects of different fractions of methanolic extract of Momordica charantia (Linn.) in alloxan induced diabetic rats. Int. J. Pharm. Sci. Res. 2, 601-627.

Kaleem, M., Asif, M., Ahmed, Q.U. and Banom B. 2006. Antidiabetic and antioxidant activity of Annona squamosa extract in streptozotocin-induced diabetic rats. Singapore Med. J. 47, 670-675.

Leeds, J.S., Forman, E.M., Morley, S., Scott, A.R., Tesfaye, S. and Sanders, D.S. 2009. Abnormal liver function tests inpatients with Type 1 diabetes mellitus: prevalence, clinical correlations and underlying pathologies. Diabet. Med. 26, 1235-1241.

Lin, Y.F., Tsai, H.L., Lee, Y.C. and Chang, S.J. 2005. Maternal vitamin E supplementation affects the antioxidant capability and oxidative status of hatching chicks. J. Nutr. 135, 24572461.

Meybodi, M.A., Afkhami-Ardekani, M. and Rashidi, M. 2008. Prevalence of abnormal serum alanine aminotransferase levels in type 2 diabetic patients in Iran. Pak. J. Biol. Sci. 11, 2274-2277.

Feshani, A.M., Kouhsari, S.M. and Mohammadi S. 2010. Vaccinium arctostaphylos, a common herbal medicine in Iran: Molecular and biochemical study of its antidiabetic effects on Alloxan-diabetic Wistar rats. J. Ethnopharmacol. 133, 67-74.

Oyedemi, S., Bradley, G. and Afolayan, A. 2011. Antidiabetic activities of aqueous stem bark extract of Strychnoshenningsii Gilg in streptozotocin-nicotinamide type 2 diabetic rats. Iranian J. Pharm. Res. 10, 221-228.

Senior, J.R. 2009. Monitoring for hepatotoxicity: what is the predictive value of liver "function" tests? Clin. Pharmacol. Ther. 85, 331-334.
Sepici-Dincel, A., Acikgoz, S., Cevik, C., Sengelen, M. and Yesilada, E. 2007. Effects of in-vivo antioxidant enzyme activities of myrtle oil in normoglycaemic and alloxan diabetic rabbits. J. Ethnopharmacol. 110, 498-503.

Simmons, R.A. 2006. Developmental origins of diabetes: the role of oxidative stress. Free Radic. Biol.Med. 40, 917-922.

Soon, Y.Y. and Tan, B.K. 2002. Evaluation of the hypoglycemic and anti-oxidant activities of Morindaofficinalis in streptozotocin-induced diabetic rats. Singapore Med. J. 43, 77-85.

Soret, M.G., Dulin, W.E., Mathews, J. and Gerritsen, G.C. 1974. Morphologic abnormalities observed inretina, pancreas, and kidney of diabetic Chinese hamsters. Diabetologia 10, $567-$ 579.

Telci, A., Cakatay, U., Salman, S., Satman, I. and Sivas, A. 2000. Oxidative protein damage in early stagein type 1 diabetic patients, Diabetes Res. Clin. Pract. 50, 213-223.

Turk, H.M., Sevinc, A., Camci, C., Cigli, A., Buyukberber, S., Savli, H. and Bayraktar, N. 2002. Plasmalipid peroxidation products and antioxidant enzyme activities in patients with type 2 diabetes mellitus. Acta Diabetol. 39, 117-122.

UK Prospective Diabetes Study (UKPDS) Group. 1998. Intensive blood-glucose control with sulphonylureas or insulin compared with conventional treatment and risk of complications in patients with type 2 diabetes (UKPDS 33). Lancet 352, 837-853.

Vincent, A.M., Russell, J.W., Low, P. and Feldman, E.L. 2004. Oxidative stress in the pathogenesis of diabetic neuropathy. Endocr. Rev. 25, 612-628.

Vozarova, B., Stefan, N., Lindsay, R.S., Saremi, A., Pratley, R.E., Bogardus, C. and Tataranni, P.A. 2002. High alanineaminotransferase is associated with decreased hepatic insulin sensitivity and predicts the development of type2 diabetes. Diabetes 51, 1889-1895.

Zannah, S., Islam, M.S., Rahman, A.F.M.T., Asaduzzaman, M., Bari, A.A.A., Ali, Y., Islam, G.J., Alam, A.H.M.K., Ali, H. and Rashid, M. 2014. Antidiabetic drugs in combination with hydroxychloroquine improve glycemic control in alloxan induced diabetic rats. Pharmacology \& Pharmacy 5, 725-735. 